\title{
General Phase Diagram of Multimodal Ordered and Disordered Lasers in Closed and Open Cavities
}

\author{
F. Antenucci, ${ }^{1,2}$ C. Conti, ${ }^{1,3}$ A. Crisanti, ${ }^{1,3}$ and L. Leuzzi ${ }^{2,1, *}$ \\ ${ }^{1}$ Dipartimento di Fisica, Università di Roma "Sapienza," Piazzale A. Moro 2, I-00185 Roma, Italy \\ ${ }^{2}$ IPCF-CNR, UOS Kerberos Roma, Piazzale A. Moro 2, I-00185 Roma, Italy \\ ${ }^{3}$ ISC-CNR, UOS Sapienza, Piazzale A. Moro 2, I-00185 Roma, Italy
}

(Received 29 June 2014; published 30 January 2015)

\begin{abstract}
We present a unified approach to the theory of multimodal laser cavities including a variable amount of structural disorder. A general mean-field theory is studied for waves in media with variable nonlinearity and randomness. Phase diagrams are reported in terms of optical power, degree of disorder, and degree of nonlinearity, tuning between closed and open cavity scenarios. In the thermodynamic limit of infinitely many modes, the theory predicts four distinct regimes: a continuous wave behavior for low power, a standard mode-locking laser regime for high power and weak disorder, a random laser for high pumped power and large disorder, and a novel intermediate regime of phase locking occurring in the presence of disorder but below the lasing threshold.
\end{abstract}

DOI: 10.1103/PhysRevLett.114.043901

PACS numbers: 42.55.Zz, 42.60.Fc, 64.70.P-, 75.50.Lk

In describing cavityless lasers with randomly placed scatterers, called random lasers (RLs) [1-11], the challenging issue is the interplay between disorder and nonlinearity. In RLs, lasing is due to stimulated amplification spatially localized in leaky stochastic resonators [1]. If disorder is dominant, the stimulated amplification of light can be hindered because of diffusion. Conversely, if structural disorder is weak, the effect on nonlinear evolution is marginal and does not modify the standard laser features. Competition occurs when wave scattering affects the degree of localization and nonlinearity couples the localized modes. Recent experimental results show that the coupling of modes changes their localizations and their spatial and spectral correlations [12-14].

We report a theoretical analysis accounting for the fact that light modes exhibit a distribution of localization lengths and an interaction determined by the overall energy. In a statistical mechanical framework, we predict specific transitions from incoherent to coherent regimes, both in the case of standard mode-locking (ML) lasers in a closed cavity and for cavityless systems with strong gain in random media. The theory is based on the hypothesis of effective equilibrium. Lasers are manifestly off equilibrium, and energy is pumped into the system to maintain population inversion and stimulated emission and, in open cavities, also to compensate for radiation losses. As power is kept constant, though, the resulting stationary regime can be described as if at equilibrium with an effective "thermal bath" whose "temperature" is related to the pumping rate and to the environment temperature $[15,16]$.

Lasing in random media displays a glassy coherent behavior. As glassy we mean that (i) a subset of modes out of an extensive ensemble of localized passive modes are activated in a nondeterministic way [17] and (ii) the whole set of activated modes behaves cooperatively and belongs to one state out of many possible ones. The system properties can be represented by a corrugated landscape composed of many valleys separated by high mountains and hidden passes. The overall coherence arises from the trapping in a metastable state in the landscape.

We identify four different phases: continuous wave (CW), phase-locked wave (PLW), standard ML laser (SML), and random lasing (RL). In previous work, mode phases were retained as the only relevant dynamic variables $[9,16]$. Here, we remove this "quenched amplitude" approximation and provide a general picture of the regimes attainable in a multimodal laser at any degree of pumping, disorder, and cavity leakage.

The complex amplitude model.-For a closed cavity, localized modes form a complete set, and the electromagnetic field $\boldsymbol{E}(\boldsymbol{r}, t)$ can be expanded in terms of normal modes $\boldsymbol{E}_{n}(\boldsymbol{r})$ with time-dependent complex amplitudes $a_{n}(t)$ [16]. In open cavities a continuous spectrum of radiation modes is also present. The contributions of radiative and localized modes can be separated by Feshbach projection onto two orthogonal subspaces [20]. This leads to an effective theory on the subspace of localized modes in which they exchange a linear effective damping coupling $[21,22]$. Radiation losses and gain are accounted for by additional linear terms, and the presence of a thermal bath is represented by the fluctuations due to the spontaneous emission. Nonlinear couplings arise from gain saturation and from the optical Kerr effect. At equilibrium with the pump mechanism, the amplitudes $a_{n}(t)$ are linked by a constraint given by the total intensity inside the system $\mathcal{E}=\epsilon N=\sum_{n=1}^{N}\left|a_{n}\right|^{2}$.

Within a semiclassical approach for light propagation and amplification in nonlinear media and including 
randomness $[9,11,16,23]$ and radiation from open cavities [21,22,24-26], a general Hamiltonian is derived in the slow amplitude approximation $[9,16,23]$

$$
\begin{gathered}
\mathcal{H}=-\Re\left[\frac{1}{2} \sum_{\vec{n}_{2}}^{1, N} J_{\vec{n}_{2}} a_{n_{1}} a_{n_{2}}^{*}+\frac{1}{4 !} \sum_{\operatorname{ML}\left(\vec{n}_{4}\right)}^{1, N} J_{\vec{n}_{4}} a_{n_{1}} a_{n_{2}}^{*} a_{n_{3}} a_{n_{4}}^{*}\right], \\
\operatorname{ML}\left(\vec{n}_{4}\right):\left|\omega_{n_{1}}-\omega_{n_{2}}+\omega_{n_{3}}-\omega_{4}\right| \lesssim \gamma
\end{gathered}
$$

where $\vec{n}_{p}=\left\{n_{1} \ldots n_{p}\right\}$ and the ML sum ranges over the sets of distinct indices $\vec{n}_{4}$ for which the so-called ML condition holds, being $\gamma$ the typical linewidth of the modes.

The coupling $J_{\vec{n}_{4}}$ represents the spatial overlap of the electromagnetic fields modulated by nonlinear $\chi^{(3)}$ susceptibility

$$
\begin{aligned}
J_{\vec{n}_{4}}= & \frac{l}{2} \prod_{j=1}^{4} \sqrt{\omega_{n_{j}}} \int_{V} d^{3} r \chi_{\vec{\alpha}_{4}}^{(3)}\left(\left\{\omega_{\vec{n}_{4}}\right\} ; \mathbf{r}\right) \\
& \times E_{n_{1}}^{\alpha_{1}}(\mathbf{r}) E_{n_{2}}^{\alpha_{2}}(\mathbf{r}) E_{n_{3}}^{\alpha_{3}}(\mathbf{r}) E_{n_{4}}^{\alpha_{4}}(\mathbf{r})
\end{aligned}
$$

with $\alpha_{j}=x, y, z$, and $\vec{\alpha}_{4}=\left\{\alpha_{1}, \alpha_{2}, \alpha_{3}, \alpha_{4}\right\}$. The linear coefficient $J_{\vec{n}_{2}}$ yields different contributions depending on medium randomness and cavity leakage,

$$
\begin{gathered}
J_{\vec{n}_{2}}=J_{\vec{n}_{2}}^{\mathrm{inh}}+J_{\vec{n}_{2}}^{\mathrm{rad}}, \\
J_{\vec{n}_{2}}^{\mathrm{inh}}=\frac{l}{2} \sqrt{\omega_{n_{1}} \omega_{n_{2}}} \int_{V} d^{3} r \epsilon_{\vec{\alpha}_{2}}(\mathbf{r}) E_{n_{1}}^{\alpha_{1}}(\mathbf{r}) E_{n_{2}}^{\alpha_{2}}(\mathbf{r}) .
\end{gathered}
$$

A nonuniform distribution of the dielectric permittivity tensor $\boldsymbol{\epsilon}(\boldsymbol{r})$ [27-30] and, possibly, of the gain yields the spatial overlap of localized eigenmodes $J^{\text {inh }}$. Besides, in the "open cavity" scenario, the linear terms also account for the presence of a continuous spectrum, and they correspond to the effective damping contribution $J^{\mathrm{rad}}[21,22]$. In the "strong cavity limit," [27] $J^{\mathrm{rad}}=0$ by construction and $J^{\text {inh }}$ is diagonal.

We build a mean-field theory in which the system is fully connected. This amounts to adopt a "narrow bandwidth" approximation for the gain profile in which $\left|\omega_{j}-\omega_{k}\right|<\gamma$, for each $j, k=1, \ldots, N[9,31-33]$. This occurs in the "dispersive" RLs with very low finesse and a sensitive narrowing of the bandwidth above threshold [2], in which many modes oscillate in a relative small bandwidth with overlapping linewidths. This implies (i) that the net gain is frequency independent, $J_{n}=g\left(\omega_{n}\right) \simeq g\left(\omega_{0}\right)=g_{0}$, and (ii) that different modes can linearly interact without changing their frequencies. Because of the latter, we will consider nonzero off-diagonal elements of the linear coupling to maintain the most general level of description. Although in cases of dominant linear coupling of spectrally distinct modes, the interaction matrix $J_{\vec{n}_{2}}$ can always be diagonalized, introducing a complete set of left and right eigenmodes, as done, e.g., in Refs. [34,35]; this is not granted for all laser systems if resonances are overlapping and if nonlinearity prevails. More specifically, in our approach, the nonlinear random coupling is treated nonperturbatively, because the complex structure of glassy RLs well above threshold cannot be probed by using perturbation theory to the linear regime.

The open cavity model can be viewed as an extension to complex variables of the so-called spherical $2+p$ model [36-40], yielding a far richer variety of physical scenarios.

Couplings may, in general, be disordered because modes display different degree and shape of localizations [41,42]. The constituents of the integrals in Eq. (2) and (4) are very difficult to calculate from first principles. The only specific form of the nonlinear susceptibility has been computed by Lamb $[43,44]$ for few-modes standard lasers, and no analogue study for RLs has been performed so far, to our knowledge. Overlap integrals in a disordered system can be regarded as a sum over many random variables. Different couplings involving a given mode are, in general, correlated [26]. Since we work in the limit of an infinite number of modes and correlations decay with the size of the system, though, we adopt as working hypothesis a Gaussian distribution for each $J_{\vec{n}_{p}}$,

$$
P\left(J_{\vec{n}_{p}}\right)=\sqrt{\frac{N^{p-1}}{2 \pi J_{p}^{2}}} \exp \left\{-\frac{N^{p-1}}{2 J_{p}^{2}}\left[J_{\vec{n}_{p}}-\frac{J_{0}^{(p)}}{N^{p-1}}\right]^{2}\right\},
$$

with $p=2,4$. We stress that, though technically more committing, considering correlated $J$ 's leads to qualitatively analogue phase diagram as well known in spin-glass systems such as, e.g., the random orthogonal model $[45,46]$. To simplify the computation and its presentation, we will take real-valued interaction couplings. This amounts, e.g., to neglect of the effect of group velocity in the diagonal linear part and the Kerr lens effect in the nonlinear term but does not change the generality of the qualitative picture. This is the most general Hamiltonian model for laser systems that one can consider. Adding further nonlinear terms $\left(J_{\vec{n}_{p}}\right.$ with $\left.p=3,5,6, \ldots\right)$ [33] does not alter the qualitative behavior at the transition from continuous wave to lasing regimes.

The external parameters.-In order to yield a comprehensive description, we introduce the degrees of nonlinearity $\alpha_{0}, \alpha$, varying in the interval $[0,1]$, and suitable interaction energy scales $J_{0}, J$, for the ordered and disordered component, respectively,

$$
\begin{gathered}
J_{0}^{(4)}=\alpha_{0} J_{0} ; \quad \alpha_{0}=\left[\frac{J_{0}^{(2)}}{J_{0}^{(4)}}+1\right]^{-1} ; \quad J_{0}=J_{0}^{(2)}+J_{0}^{(4)}, \\
J_{4}=\alpha J ; \quad \alpha=\left[\frac{J_{2}}{J_{4}}+1\right]^{-1} ; \quad J=J_{2}+J_{4} .
\end{gathered}
$$

The "degree of disorder" of a given system with coupling parameter scales $J, J_{0}$ is, then, defined as $R_{J}=J / J_{0}$. 
The average energy per mode $\epsilon$ is related to the so-called "pumping rate" $\mathcal{P}$ induced by the pumping laser source in the RL or proportional to the optical power in the cavity for the standard laser. In the present Letter it is defined as $\mathcal{P} \equiv$ $\epsilon \sqrt{J_{0} / k_{B} T}=\epsilon \sqrt{\beta J_{0}}$ where $T$ is the heat-bath temperature. It encodes the experimental evidence that decreasing the temperature [47] or increasing the total power [48] yields qualitatively similar behaviors. As mentioned in the introduction, $1 / \mathcal{P}^{2} \propto T / \epsilon^{2}$ plays the role of the temperature of the effective thermal bath with which the system is at equilibrium. The proportionality factor $J_{0}$ is a material-dependent parameter function of the central angular frequency $\omega_{0}$, cf. Eq. (2), and it is volume independent.

To summarize, the parameters of interest are

\begin{tabular}{ll}
\hline Optical power per mode & \multicolumn{1}{c}{$\epsilon$} \\
\hline Heat-bath thermal energy & $k_{B} T=1 / \beta$ \\
Cumulative coupling average & $J_{0}=J_{0}^{(2)}+J_{0}^{(4)}$ \\
Cumulative mean square disp. & $J=J_{2}+J_{4}$ \\
Pumping rate & $\mathcal{P}=\epsilon \sqrt{\beta J_{0}}$ \\
Disorder degree & $R_{J}=J / J_{0}$ \\
Nonlinearity degree (ordered) & $\alpha_{0}=J_{0}^{(4)} / J_{0}$ \\
Nonlinearity degree (disordered) & $\alpha=J_{4} / J$.
\end{tabular}

We will consider here $\alpha=\alpha_{0}$ for simplicity, but cases with different degrees of nonlinearity in ordered and disordered contributions can be also analyzed.

Statistical mechanics with replicas.-We study the model by means of the replica trick [49]. This enables us to calculate the average free energy $f$ in the one-step replica symmetry-breaking (1RSB) ansatz as a function of generalized order parameters, as detailed in the Supplemental Material [50]. We find a series of order parameters describing the physical regimes: (i) the intensity coherence of activated modes $m$, (ii) the phase coherence $r_{d}$, (iii) and (iv) the overlap parameters $q_{0,1}$, and (v) the RSB parameter $x$. The latter three specify degree and kind of glassiness. The free energy reads

$$
\begin{aligned}
2 \beta f\left(q_{0}, q_{1}, r_{d}, m\right)= & 2 \beta f_{0}+(1-x) w\left(q_{1}, q_{1}\right) \\
& +x w\left(q_{0}, q_{0}\right)-w\left(r_{d}, 1\right) \\
& -2 k(m)-\ln \left(1-r_{d}\right) \\
& -\ln \mathcal{X}_{1}-\frac{1}{x} \ln \frac{\mathcal{X}_{0}}{\mathcal{X}_{1}}-\frac{2 q_{0}-m^{2}}{\mathcal{X}_{0}}
\end{aligned}
$$

with

$$
\begin{gathered}
\mathcal{X}_{1} \equiv 1+r_{d}-2 q_{1} ; \quad \mathcal{X}_{0} \equiv \mathcal{X}_{1}+2 x\left(q_{1}-q_{0}\right), \\
w(t, u) \equiv \xi_{2}\left(t^{2}+u^{2}\right)+\frac{\xi_{4}}{2}\left(t^{4}+u^{4}+4 t^{2} u^{2}\right), \\
k(m) \equiv k_{2}|m|^{2}+k_{4}|m|^{4}, \\
\xi_{2}=\frac{\beta^{2} \epsilon^{2}}{4} J_{2}^{2} ; \quad \xi_{4}=\frac{\beta^{2} \epsilon^{4}}{6} J_{4}^{2},
\end{gathered}
$$

$$
k_{2}=\frac{\beta \epsilon}{4} J_{0}^{(2)} ; \quad k_{4}=\frac{\beta \epsilon^{2}}{96} J_{0}^{(4)} .
$$

The self-consistency equations for the order parameters are given in the Supplemental Material [50]. These determine all relevant thermodynamic phases, as displayed in the phase diagrams reported in Fig. 1.

Continuous wave regime: For small optical power, all modes oscillate independently in a $\mathrm{CW}$ incoherent noisy
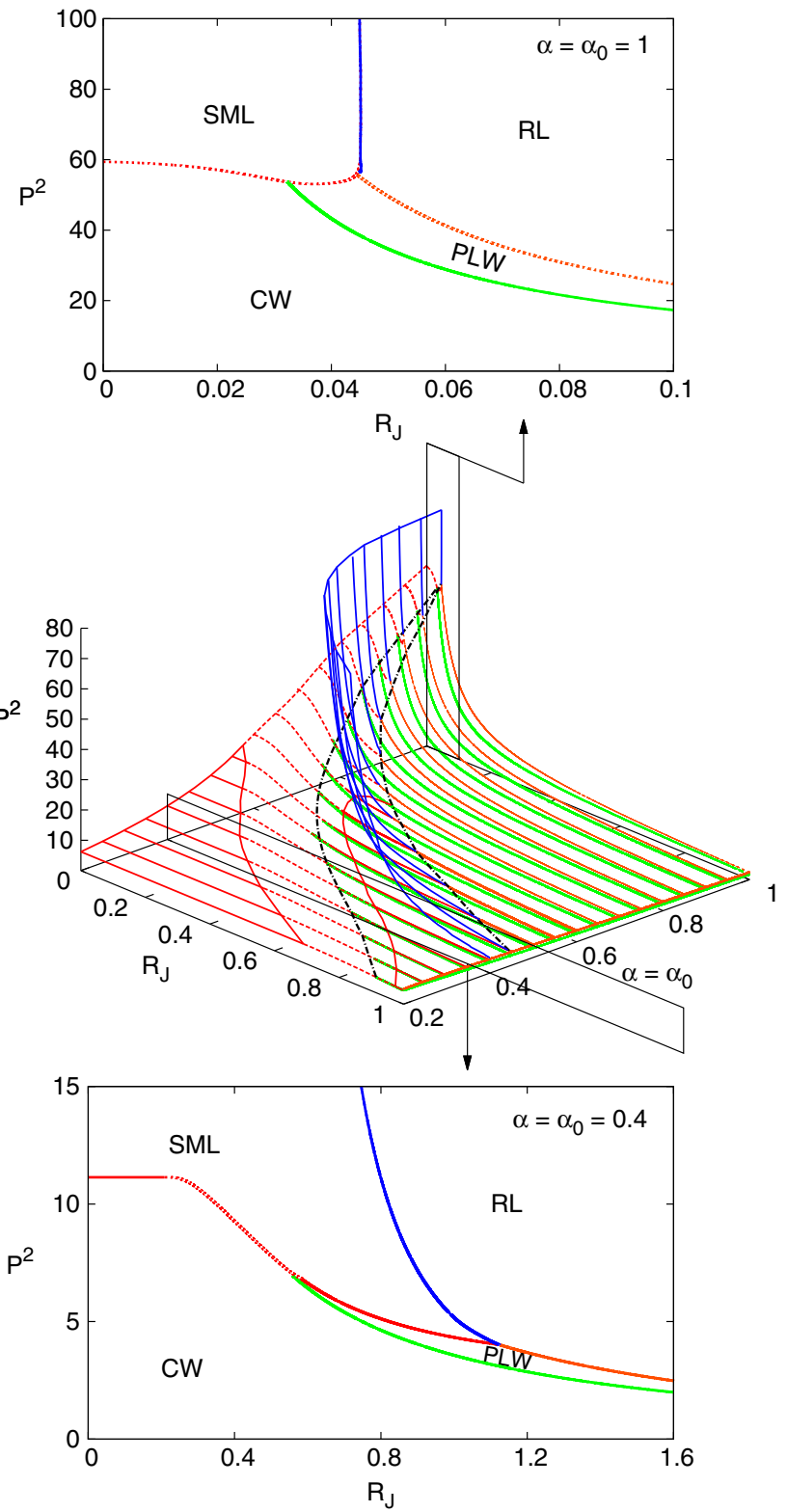

FIG. 1 (color online). Phase diagrams in the $\left(\mathcal{P}^{2}, R_{J}, \alpha=\alpha_{0}\right)$ space. For low disorder, only the SML and CW phases occur, varying pumping and degree of linearity. As disorder increases, the intermediate PLW phase arises between CW and SML. For strong disorder, RL replaces SML above the pumping threshold line. For any $R_{J}$, for low $\alpha_{0}$ the transition driven by $\mathcal{P}$ is continuous in the order parameters, whereas for high nonlinearity $\left(\alpha>\alpha_{\mathrm{nl}}=0.6297\right)$ it is discontinuous. Insets: phase diagrams in the $\left(\mathcal{P}^{2}, R_{J}\right)$ plane for closed $\left(\alpha=\alpha_{0}=1\right)$ and open $\left(\alpha=\alpha_{0}=0.4\right)$ cavities. 
regime and the energy equally fluctuates among all passive modes. At low $\mathcal{P}$, for any degree of quenched disorder $R_{J}$ and nonlinearity $\alpha$, all parameters are $q_{01}=m=r_{d}=0$ ( $x$ is irrelevant when $q_{0}=q_{1}$ ).

Phase locking wave regime: For $R_{J}>0$, on increasing $\mathcal{P}$ the system undergoes a transition to a thermodynamic phase in which the mode phases lock on one given value, without stimulated amplification. Considering complex amplitudes as planar continuous spherical spins, this corresponds to all spins pointing in the same direction, though their intensity is freely oscillating. This phase has no counterpart in statistical mechanical models studied so far. Phase coherence $r_{d}$ is nonzero, whereas $q_{0,1}=m=0$. With an increasing nonlinearity $\alpha$, PLW occurs at a lower and lower pumping rate $\mathcal{P}$.

Standard mode-locking laser: For large $\mathcal{P}$ and $R_{J}=0$, a localization transition occurs and the intensity is shared by activated modes, all of them oscillating coherently. This corresponds to standard passive ML lasers [55], where a passive transition in $\mathcal{P}$ is predicted as a paramagneticferromagnetic transition in Ref. [56,57]. We further find that the CW/SML transition takes place also in the presence of a limited amount of disorder $R_{J} \gtrsim 0$. In this regime $m \neq 0$ : modes are coherent in intensity and stimulated amplification occurs.

Random lasing: For high pumping rate and strong disorder, synchronous oscillations are frustrated, resulting in a glassy phase representing the RL regime. Modes are all coherent in phase $\left(r_{d} \neq 0\right)$ but not in intensity $(m=0)$. In the glass phase, RSB occurs: $q_{1}>q_{0}=0$ and $x \neq 0$; see the Supplemental Material [50] for details.

Phase diagram.-In Fig. 1 we show the $\mathcal{P}^{2}, \alpha, R_{J}$ diagram in the main three-dimensional plot. In the top inset the closed cavity projection for $\alpha=1$ is displayed, and in the bottom inset an open cavity instance $\alpha=0.4$ is displayed.

For low degree of disorder $R_{J}$, there is a threshold between a CW (or PLW) phase and a SML; for large $R_{J}$ the threshold is to a RL. The CW/SML threshold line is plotted as a solid (continuous transition) or a dotted (discontinuous transition) dark (red) line, and it occurs for $R_{J} \gtrsim 0$. The locus of tricritical (SML,CW,PWL) transition points is plotted as a black dashed-dotted line.

As $R_{J}$ is still small but a bit larger than the tricritical point, on increasing the pumping one first has a CW/PWL transition denoted by a continuous light gray (green) line and then a PWL/SML. The latter can be continuous (solid dark-gray/red line) or discontinuous (dotted line).

For larger $R_{J}$ we exclusively observe PWL/RL transition. The nature of the PWL/RL transition depends on the degree of nonlinearity; it occurs both continuously, for $\alpha<\alpha_{\mathrm{nl}}$, and discontinuously, for $\alpha>\alpha_{\mathrm{nl}}$ (Supplemental Material [50]).

Supposing, furthermore, that in an optically active material the degree of disorder $R_{J}$ could be continuously changed, the phase diagram also predicts a transition between SML and

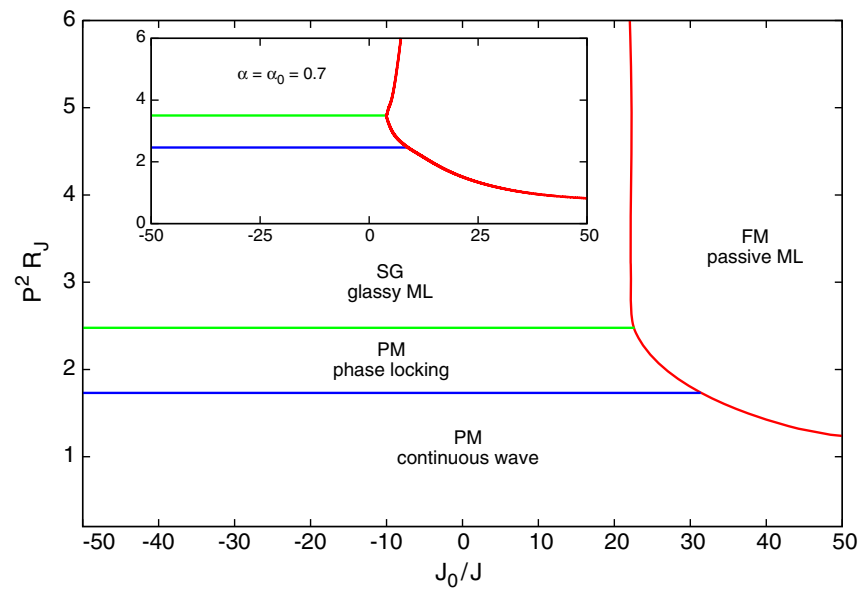

FIG. 2 (color online). Phase diagram in $\mathcal{P}^{2} R_{J}=\beta \epsilon^{2} J$ vs $J_{0} / J=1 / R_{J}$ for closed cavity $\alpha=\alpha_{0}=1$ for $J_{0} / J$ ranging from negative to positive. Inset: open cavity with $\alpha=0.7$.

RL for pumping above threshold, represented by the nearly vertical solid (blue) transition lines (Fig. 1). The locus of the tricritical points RL/SML/PWL is plotted as a second black dashed-dotted line in the main plot.

Mode locking without saturable absorber.-A key point in the present study is that the transition from continuous wave to standard passive mode-locking ( $\mathrm{CW} \rightarrow \mathrm{SML}$ ) only occurs for a strictly positive value of the coupling coefficient $J_{0}$, as shown in Figs. 1 and 2. This formally corresponds to the presence of a saturable absorber in the cavity [16]. In RLs such a device is not present, and hence, the occurrence of this lasing transition is not to be taken for granted. However, in Fig. 1 it is shown that on starting from a standard laser supporting passive mode locking and increasing the disorder, the CW/SML transition acquires the character of a glassy mode-locking CW/RL transition. This is also present for $J_{0}<0$, as explicitly shown in Fig. 2. It is far from trivial that the latter mode-locking transition, ruled out for ordered lasers without a saturable absorber $\left(J \ll J_{0}\right)$, spontaneously occurs as an effect of the quenched disorder, i.e., for large enough $R_{J}$. The physical origin is the mode coupling due to the open cavity configuration.

Conclusions.-We developed a theory for light wave systems in optically active random media with narrow bandwidth and in which all activated mode localizations spatially overlap each other. The theory is based on the most general Hamiltonian for an open system, including nonlinearity and coupling to a thermal bath, effectively representing the energy transfer to the system by an external pumping keeping the total power constant. This corresponds to a mean field fully connected disordered system in statistical mechanics. We derive the most general phase diagram, ranging from closed cavities that correspond to a standard laser, to completely open cavities, representing the random lasers. The resulting picture, given in terms of order parameters for correlations among amplitudes, phases, and their cross correlation, furnishes a number of different equilibrium phases affected by nonlinearity and disorder. 
These include standard passive-mode locking in standard lasers and different coherent regimes attainable in random lasers. The reported results open the way to further investigations, as the study of open quantum systems, testing theoretical models for the statistical mechanics of disordered systems, employing variable coherence sources for applications in spectroscopy and microscopy, and developing novel techniques for mode locking and ultrashort pulse generation not requiring saturable absorbers.

The research leading to these results received funding from the People Programme (Marie Curie Actions) of the European Union's Seventh Framework Programme FP7/ 2007-2013 under REA Grant agreement No. 290038, NETADIS project, from the European Research Council through ERC Grant agreement No. 247328, CriPheRaSy project, and from the Italian MIUR under the Basic Research Investigation Fund FIRB2008 program, Grant No. RBFR08M3P4, and under the PRIN2010 program, Grant code No. 2010HXAW77-008.

*luca.leuzzi@cnr.it

[1] V. S. Lethokov, Sov. Phys. JETP 26, 835 (1968).

[2] N. M. Lawandy, R. M. Balachandran, A. S. L. Gomes, and E. Sauvain, Nature (London) 368, 436 (1994).

[3] H. Cao, Y. G. Zhao, S. T. Ho, E. W. Seelig, Q. H. Wang, and R. P. H. Chang, Phys. Rev. Lett. 82, 2278 (1999).

[4] P. Sebbah and C. Vanneste, Phys. Rev. B 66, 144202 (2002).

[5] H. Cao, J. Phys. A 38, 10497 (2005).

[6] H. E. Tureci, A. D. Stone, and B. Collier, Phys. Rev. A 74, 043822 (2006).

[7] H. E. Tureci, L. Ge, S. Rotter, and A. D. Stone, Science 320, 643 (2008).

[8] D. S. Wiersma, Nat. Phys. 4, 359 (2008).

[9] L. Leuzzi, C. Conti, V. Folli, L. Angelani, and G. Ruocco, Phys. Rev. Lett. 102, 083901 (2009).

[10] S. K. Turitsyn, S. A. Babin, A. E. El-Taher, P. Harper, D. V. Churkin, S. I. Kablukov, J. D. Ania-Castanon, V. Karalekas, and E. V. Podivilov, Nat. Photonics 4, 231 (2010).

[11] O. Zaitsev and L. Deych, J. Opt. 12, 024001 (2010).

[12] M. Leonetti, C. Conti, and C. Lopez, Nat. Commun. 4, 1740 (2013).

[13] M. Leonetti and C. Lopez, Appl. Phys. Lett. 102, 071105 (2013).

[14] M. Leonetti, C. Conti, and C. Lopez, Light Sci. Appl. 2, e88 (2013).

[15] A. Gordon and B. Fischer, Phys. Rev. Lett. 89, 103901 (2002).

[16] C. Conti and L. Leuzzi, Phys. Rev. B 83, 134204 (2011).

[17] Nondeterministic does not mean chaotic, since chaos is deterministic. It is an apart dynamic phenomenon occurring in laser systems [18], not affecting the presence or absence of glassiness, as, e.g., shown in Ref. [19]. Chaos is not a necessary nor a sufficient feature for RLs.

[18] C. Weiss and R. Vilaseca, Dynamics of Lasers (VCH, Weinheim, Germany, 1991).

[19] A. Crisanti, M. Falcioni, and A. Vulpiani, Phys. Rev. Lett. 76, 612 (1996).
[20] H. Feshbach, Ann. Phys. (N.Y.) 19, 287 (1962).

[21] G. Hackenbroich, C. Viviescas, and F. Haake, Phys. Rev. A 68, 063805 (2003).

[22] C. Viviescas and G. Hackenbroich, Phys. Rev. A 67, 013805 (2003).

[23] L. Angelani, C. Conti, G. Ruocco, and F. Zamponi, Phys. Rev. B 74, 104207 (2006).

[24] G. Hackenbroich, C. Viviescas, and F. Haake, Phys. Rev. Lett. 89, 083902 (2002).

[25] G. Hackenbroich, J. Phys. A 38, 10537 (2005).

[26] O. Zaitsev, L. Deych, and V. Shuvayev, Phys. Rev. Lett. 102, 043906 (2009).

[27] L. I. Deych, Phys. Rev. Lett. 95, 043902 (2005).

[28] L. Ge, Y. D. Chong, and A. D. Stone, Phys. Rev. A 82, 063824 (2010).

[29] A. Cerjan, Y. Chong, L. Ge, and A. Douglas Stone, Opt. Express 20, 474 (2012).

[30] Y. D. Chong and A. D. Stone, Phys. Rev. Lett. 109, 063902 (2012).

[31] P. Meystre and M. Sargent III, Elements of Quantum Optics (Springer, New York, 1998).

[32] L. Angelani, C. Conti, G. Ruocco, and F. Zamponi, Phys. Rev. Lett. 96, 065702 (2006).

[33] O. Zaitsev and L. Deych, Phys. Rev. A 81, 023822 (2010).

[34] O. Zaitsev, Phys. Rev. A 74, 063803 (2006).

[35] O. Zaitsev, Phys. Rev. A 76, 043842 (2007).

[36] T. M. Nieuwenhuizen, Phys. Rev. Lett. 74, 4289 (1995).

[37] A. Crisanti and L. Leuzzi, Phys. Rev. Lett. 93, 217203 (2004).

[38] A. Crisanti and L. Leuzzi, Phys. Rev. B 73, 014412 (2006).

[39] A. Crisanti and L. Leuzzi, Phys. Rev. B 75, 144301 (2007).

[40] A. Crisanti and L. Leuzzi, Nucl. Phys. B870, 176 (2013).

[41] C. Conti and A. Fratalocchi, Nat. Phys. 4, 794 (2008).

[42] J. Fallert, R. J. B. Dietz, J. Sartor, D. Schneider, C. Klingshirn, and H. Kalt, Nat. Photonics 3, 279 (2009).

[43] W. E. Lamb, Phys. Rev. 134, A1429 (1964).

[44] M. Sargent III, M. O'Scully, and W. E. Lamb, Laser Physics (Addison Wesley Publishing Company, Reading, MA, 1978).

[45] E. Marinari, G. Parisi, and F. Ritort, J. Phys. A 27, 7615 (1994).

[46] G. Parisi and M. Potters, J. Phys. A 28, 5267 (1995).

[47] D. S. Wiersma and S. Cavalieri, Nature (London) 414, 708 (2001).

[48] M. Leonetti and C. Conti, J. Opt. Soc. Am. B 27, 1446 (2010).

[49] S. Edwards and P. Anderson, J. Phys. F 5, 965 (1975).

[50] See the Supplemental Material at http://link.aps.org/ supplemental/10.1103/PhysRevLett.114.043901 [brief description], which includes Refs. [51-54] for the technical details of replica theory calculation.

[51] E. Gardner, Nucl. Phys. B257, 747 (1985).

[52] A. Crisanti and H.-J. Sommers, Z. Phys. B 87, 341 (1992).

[53] A. Crisanti and L. Leuzzi, Phys. Rev. B 70, 014409 (2004).

[54] G. Parisi, J. Phys. A 13, L115 (1980).

[55] H. A. Haus, IEEE J. Quantum Electron. 6, 1173 (2000).

[56] A. Gordon and B. Fischer, Opt. Commun. 223, 151 (2003).

[57] The $R_{J}=0$ limit confirms previous estimates. To quantitatively compare to Ref. [56], one has to rescale the parameters as $T / \gamma_{s} P_{0}^{2}=8 / \mathcal{P}^{2}, P_{0}=\epsilon, \gamma_{s}=J_{0} / 8$. For quenched amplitude we have $8 / \mathcal{P}^{2}=0.1828$, and for free amplitude we find $8 / \mathcal{P}^{2}=0.1357$, both compatible with Ref. [56]. 